\title{
Arbeit und Organisation im digitalen Wandel
}

\author{
Neu erschienen: Klaus J. Zink (Hrsg.): Arbeit und Organisation im digitalen Wandel, Nomos \\ Verlagsgesellschaft, Baden-Baden 2019, 320 S., brosch., 59,- €. ISBN 978-3-8487-5755-8. \\ nomos-shop.de/41287
}

\author{
Yvonne Ferreira ${ }^{1}$ \\ Online publiziert: 19. September 2019 \\ ○) Springer-Verlag GmbH Deutschland, ein Teil von Springer Nature 2019
}

Digitalisierung ist kein neues Phänomen, aber ihre derzeitige Entwicklungsstufe, in der vor allen die Künstliche Intelligenz (KI) eine wesentliche Rolle spielt, führt zu deutlich neuen Herausforderungen für Gesellschaft und Arbeitswelt. Das Buch von Klaus J. Zink beleuchtet den derzeitigen Stand der Entwicklung und zeigt Szenarien einer Zukunft der Arbeit auf.

Hierzu wird zu Beginn auf die Veränderungen im Kontext der Arbeit eingegangen, die oftmals in Anlehnung an das deutsche Schlagwort „Industrie 4.0“ unter dem Begriff „Arbeit 4.0“ zusammengefasst sind. Arbeit und Globalisierung wird hier genauso thematisiert wie Arbeit und neue Technologien. Obwohl es neue Technologien schon immer gab, geht es in dieser Entwicklungsstufe vor allem um disruptive Technologien wie z.B. den 3-D-Druck, der viele konventionelle Herstellungsmethoden ersetzen und zu völlig neuen Geschäftsmodellen führen kann. In den anschließenden Kapiteln stehen dann die verschiedenen Möglichkeiten der Digitalisierung in den Bereichen Industrie, Dienstleistungen und Handwerk sowie den Funktionen bzw. den Prozessen (Innovation, Produktion, Beschaffung/ Einkauf, Marketing/Vertrieb, Personal) im Mittelpunkt der Betrachtung. Das bleibt nicht ohne Auswirkungen auf die Unternehmensorganisation und damit die Arbeit(sinhalte), was zu tiefgreifenden Veränderungen führen kann. Daher werden potentielle Auswirkungen auf Organisation und

\section{Prof. Dr. habil. Yvonne Ferreira}

zfa-redaktion@zfa-online.de

1 Zeitschrift für Arbeitswissenschaft, Frankfurt, Deutschland
Führung erörtert. Agile Organisationen, fluide Organisationen und Caring Companies sind ebenso Gegenstand wie Netzwerke und Crowdsourcing (in Form von Crowdwork und Work-on-Demand via Apps). Die Konsequenzen dieser neuen Organisationsformen (aber auch neuer Geschäftsmodelle) auf die Führung sind ein weiteres Thema, wobei auch die die Gestaltung von nachhaltigen Arbeitssystemen als Führungsaufgabe betrachtet wird.

Da neue Formen von Organisation und Arbeit nicht selten an ungeeigneten Transformationsprozessen scheitern, wird diesem Themenfeld ein eigenes Kapitel gewidmet. Die Einführung neuer Technologien muss allerdings auch im Kontext des gesellschaftlichen Umfeldes (z.B. des demografischen Wandels) gesehen werden, was wiederum $\mathrm{zu}$ Anforderungen an eine nachhaltige Gestaltung der Arbeit führt. In einem abschließenden Kapitel werden potentielle Auswirkungen auf den Arbeitsmarkt und die Gesellschaft insgesamt diskutiert. Dabei geht es sowohl um die verfügbaren Arbeitsmarktprognosen, als auch um Sozialversicherungssysteme und bedingungsloses Grundeinkommen.

Das Buch liefert einen guten Überblick zum Stand und den Szenarien der Digitalisierung aus einer arbeitswissenschaftlichen Perspektive und ist für jeden interessant, der sich einen aktuellen Überblick über die Veränderung in der Arbeitswelt verschaffen will. Das Buch endet mit der Feststellung: „Wir wissen noch nicht genau, was wann in welcher Form kommen wird, aber wir können es gestalten. Insofern besteht kein Grund zum Pessimismus, sondern ein Anlass zur Aufbruchstimmung, die Zukunft der Arbeit als Gestaltungsaufgabe anzugehen." Dem kann man sich nur anschließen. 